\title{
Increased Adhesion Force Due to Increased Conductivity in PEDOT:PSS/PEO Spun-coated Nanofilm
}

\author{
Raymond Christopher Setiawan, D.Y. Li \\ Department of Chemical and Materials Engineering \\ University of Alberta \\ Edmonton, AB Canada T6G 2R3 \\ rsetiawa@ualberta.ca; dongyang@ualberta.ca
}

\begin{abstract}
We propose a novel method to tune the interfacial adhesive force $\left(\mathrm{F}_{\mathrm{ad}}\right)$ between PEDOT:PSS/PEO organic electroconductive polymer and $\mathrm{Si}_{3} \mathrm{~N}_{4}$ (a ceramic) by decreasing the electron work function (EWF) and the electrical resistivity of the PEDOT:PSS/PEO nanosheet. By using the known method of preferential solvation using polar aprotic solvent (DMSO), we were able rearrange the spatial orientation of PSSH backbone chain in the polymer and increase the Density of States (DOS) and the electron hopping length ( $\left.\xi^{\prime}\right)$ of the polymer, thus increasing its conductivity. As the material becomes more conductive, the formation of a dipole layer between the Schottky Diode system of PEDOT:PSS/PEO and $\mathrm{Si}_{3} \mathrm{~N}_{4}$ is facilitated. This leads to the increased interaction between the two materials, raising the adhesive force by $35.8 \%$ with only $5 \% \mathrm{w} / \mathrm{w}$ DMSO addition to the polymer. This relatively simple treatment of PEDOT:PSS results in an interfacial adhesion or bonding strength. This approach is general, which would be applicable for enhancing interfacial bonding between two different types of material without atomic diffusion involved.
\end{abstract}

Keywords: DMSO treatment, adhesive force, electron work function, sheet resistivity, conductive organic polymer

\section{Introduction}

PEDOT:PSS has been studied quite extensively, consequently PEDOT:PSS has found itself in a myriad of application such as in solar panel or as a conductive biomaterial (sensors, etc) [1, 4]. PEDOT:PSS is also very biocompatible with a range of stem cells; therefore it has been posed as a very versatile material [6]. Pristine PEDOT:PSS is not very conductive, but it has been known that treating it with appropriate solvent (i.e. DMSO, sorbitol) can increase its conductivity manifolds [5]. However, PEDOT:PSS usage is still undermined by its inability to form good adhesion with applied surfaces or substrates [1]. In this paper we demonstrate a phenomenon of increasing the adhesion behaviour of PEDOT:PSS by virtue of increasing its sheet conductivity only, where we also have found that this also leads to subsequently decrease in electron work function (EWF) of the material. Such variations in the properties result in an increase in the interfacial adherence, which may help widen the usage of PEDOT:PSS in many applications.

\section{Methods}

PEDOT:PSS was mixed with PEO and spun-coat onto a non-conductive basal substrate. Appropriate amount of DMSO was added to enhance the conductivity of the nanofilm. The effect of the added DMSO on the surface adhesion behaviour was analysed with Atomic Force Microscopy (Adhesive force curve was obtained by using quantitative mechanical probing module). Furthermore, nano/micro-indentation tests were performed to determine if the DMSO addition affected the mechanical strength of the polymeric sheet. The conductivities of the PEDOT:PSS sheets with and without the DMSO addition were measured using the Four-Point Probe method. The electron work function was measured using a Kelvin Nano-probe.

\section{Results and Discussion}

Results of the study showed that with $5 \%$ w/w DMSO co-solvation treatment, the sheet's resistivity was decreased the value of pristine PEDOT:PSS from $1.72 \times 105 \Omega / \mathrm{sqcm}$ to $706 \Omega / \mathrm{sqcm}$ as DMSO was added, which is in line with previous studies [1,3]. Along with the decrease in electrical resistivity, the EWF of the polymeric sheet was also decreased by $34.6 \%$ hence increasing the freedom of electrons. The preceding two factors facilitate the formation of a dipole layer 
between the PEDOT:PSS/PEO and AFM's $\mathrm{Si}_{3} \mathrm{~N}_{4}$ probe, since 1) the increase in the difference in EWF between the polymer and $\mathrm{Si}_{3} \mathrm{~N}_{4}$ is raised, corresponding to an increased driving force for establishing the dipole layer at interface, and 2) more electrons are able to move towards the surface or interface between the polymeric sheet and the AFM's $\mathrm{Si}_{3} \mathrm{~N}_{4}$ probe. This leads to the increased interaction between the polymeric sheet and the $\mathrm{Si}_{3} \mathrm{~N}_{4}$ probe through the interfacial dipole-dipole interaction, leading to an increase in the adhesive force with the maximum value of $35.8 \%$ as only $5 \% \mathrm{w} / \mathrm{w}$ DMSO added to the polymer. The nano/micro-indentation tests showed that the DMSO addition did not alter Young's modulus and hardness of the polymeric sheet. Thus, the increased adhesive force should be solely contributed by the altered electrical properties of the polymeric material.

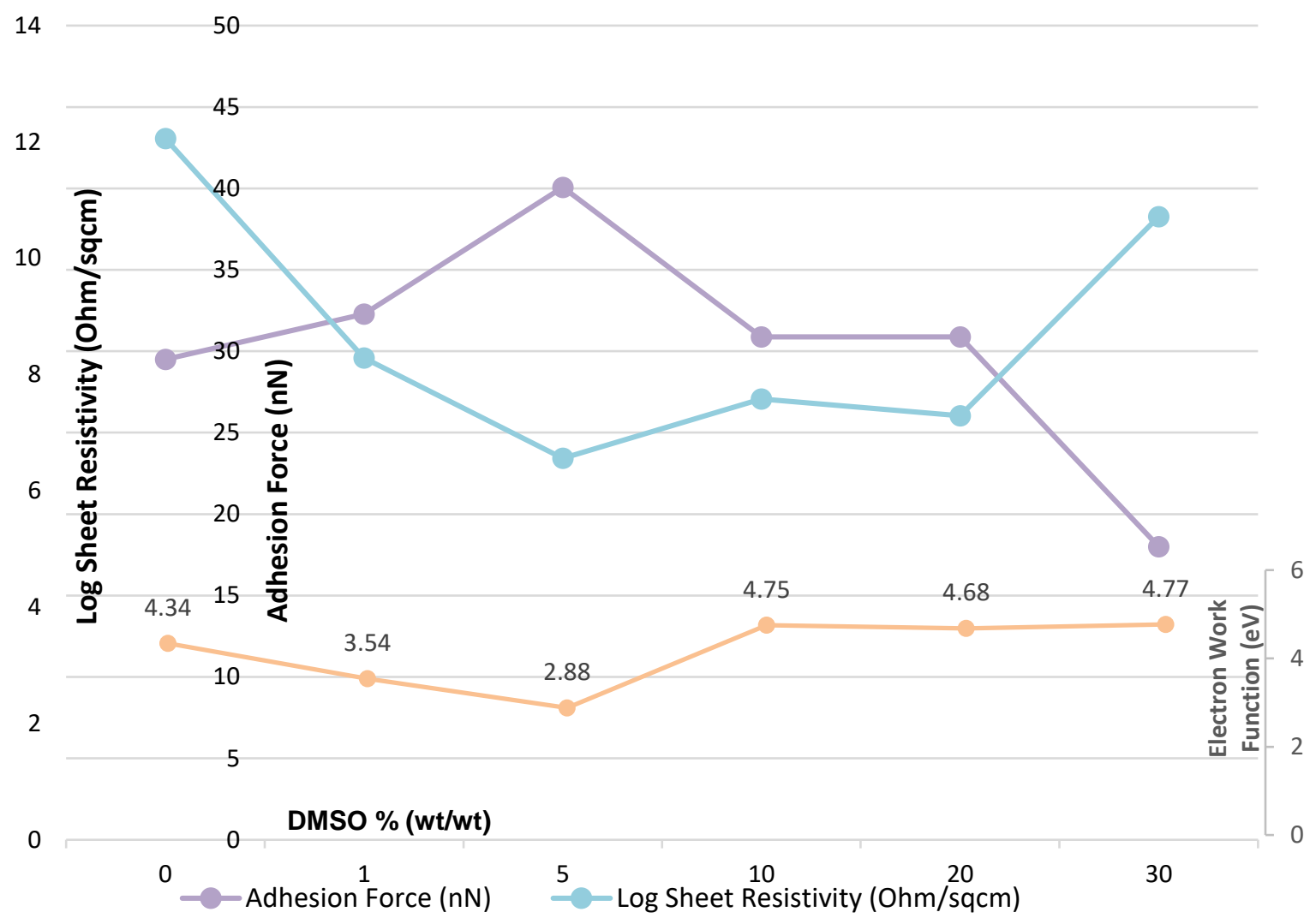

Figure 1. The profile of log sheet resistivity, interfacial adhesive force between the PEDOT:PSS/PEO and $\mathrm{Si}_{3} \mathrm{~N}_{4}$, and electron work function versus DMSO\% (wt/wt).

Fig.2 presents optical microscope images of PEDOT:PSS/PEO with and without DMSO additions. As shown, there are no significant alterations in morphology of the pristine PEDOT:PSS/PEO sheet and those with DMSO, except for the one containing 30\% (wt/wt) DMSO addition. This excessive amount of DMSO may decrease the fraction of PEDOT:PSS, thus reducing its ability to form a continuous nanofibers (therefore producing a 'blob' of isolated bubbles) 

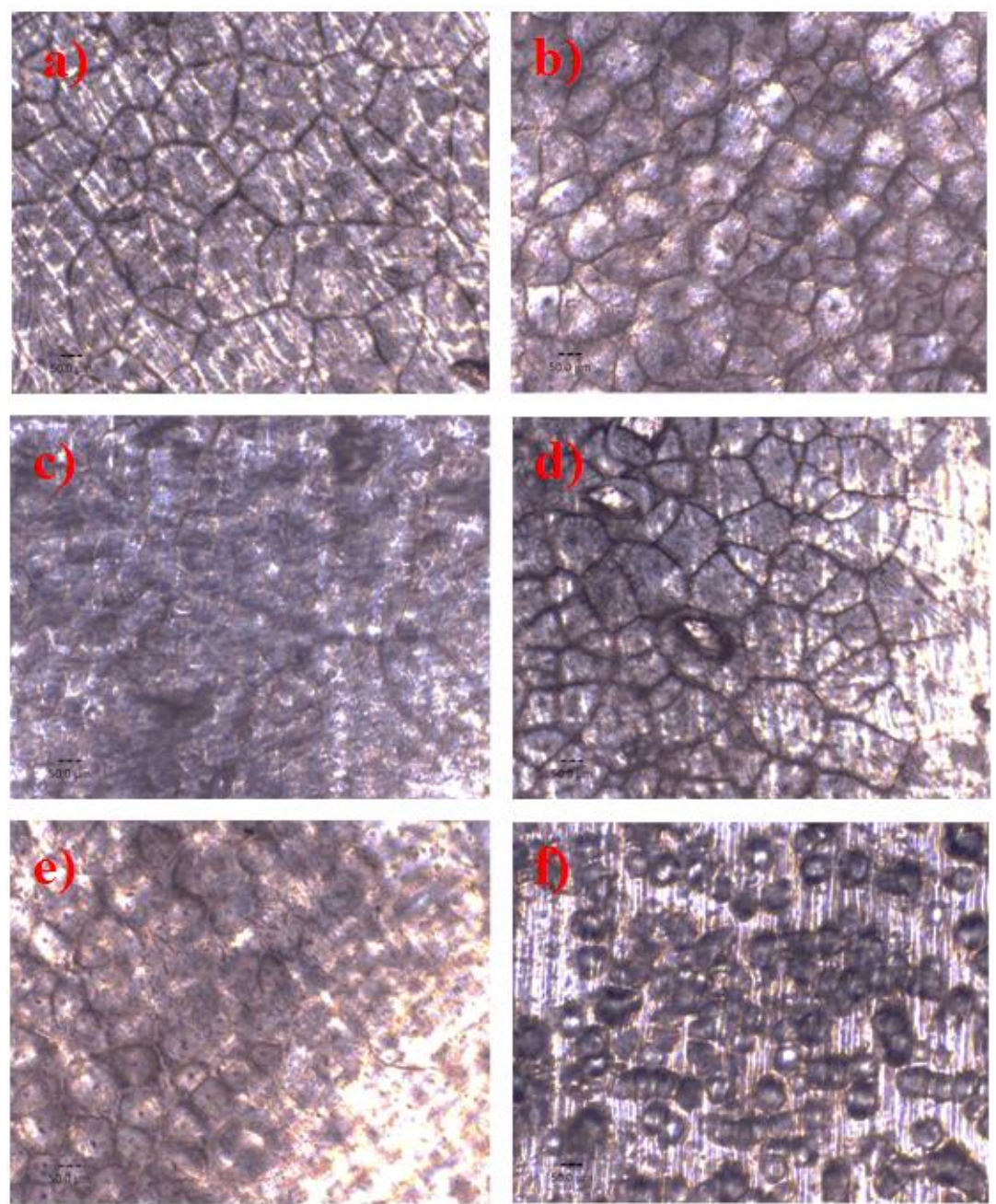

Figure 2. Optical microscope images of PEDOT:PSS/PEO sheets: a) Pristine; b) $1 \mathrm{wt} \%$ DMSO; c) $5 \mathrm{wt} \%$ DMSO; d) $10 \mathrm{wt} \%$ DMSO; e) $20 \mathrm{wt} \%$ DMSO; f) $30 \mathrm{wt} \%$ DMSO
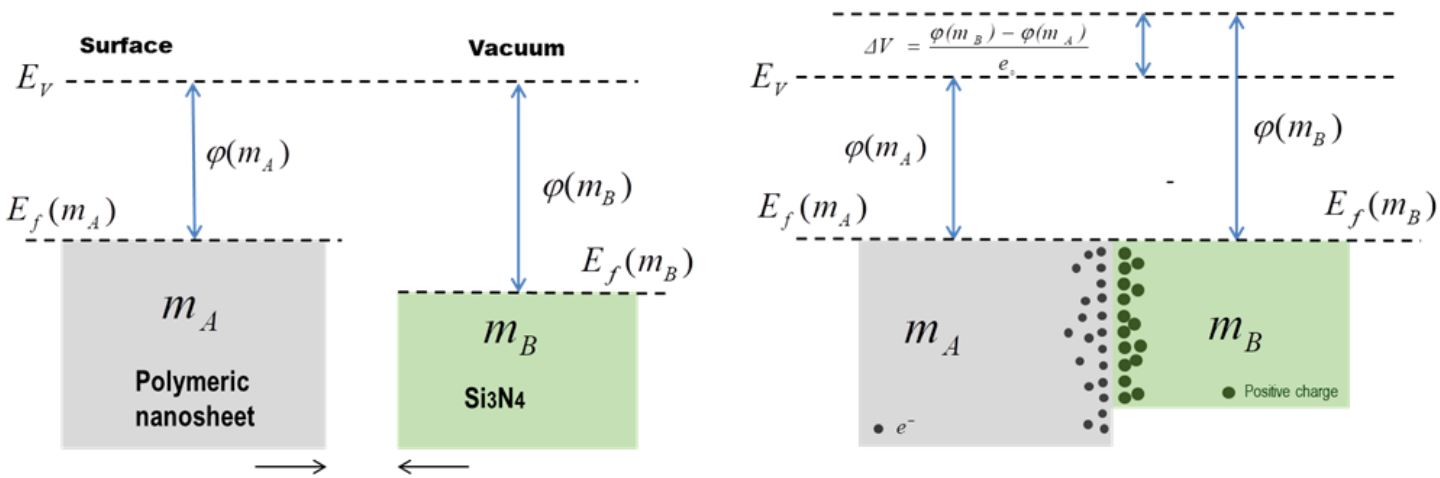

$A$ and $B$ are brought into contact

Figure 3. (Left) Two surfaces not in contact with each other are not able to form dipole layer; (Right) When in contact, a dipole layer is developed due to the difference in EWF between the two materials in contact [2].

The phenomena can be elaborated using Fig.3: when the two materials having different EWFs approach each other, their Fermi energy levels must be equal in order to reach equilibrium, which leads to a contact potential different, $\Delta V$, due 
to the difference in work function between the two materials. As a result, electrons will move towards the interface to form a dipole layer to balance the contact potential difference. This dipole layer results in electrostatic interaction, leading to the interfacial adhesion or bonding. However, if the electrons are localized, the development of the dipole layer is difficult, since only very limited charges can be induced to move locally. Thus, developing an effective dipole layer that can effectively bonding two material in contact requires sufficiently large difference in work function and certain freedom of charges. In this study, we demonstrate that using the DMSO addition to increase the electron freedom with lowered work function does increase the interfacial adhesive force between the polymeric material and $\mathrm{Si}_{3} \mathrm{~N}_{4}$.

\section{Conclusions}

The interfacial adhesive $\left(\mathrm{F}_{\mathrm{ad}}\right)$ between the electroconductive polymer and ceramic without atomic diffusion involved is dependent on two main factors: 1) the difference in EWF which generates a driving force for charge relocation, forming an interfacial dipole layer; and 2) the charge mobility or namely electrical conductivity helps the charge relocation.

\section{Acknowledgements}

The authors are grateful for support from Natural Science and Engineering Research Council (NSERC) Canada, NanoFab at the University of Alberta, Weir Minerals and Camber Tech. Corp.

\section{References}

[1] Xia Yijie, Ouyang Jianyong, "PEDOT:PSS films with significantly enhanced conductivities induced by preferential solvation with cosolvents and their application in polymer photovoltaic cells," J. Mater. Chem. 2011, vol. 21, pp. 4927-4936.

[2] Li DY, Guo Liqiu, Li Lei, Lu Hao, Electron work function - a probe for interfacial diagnosis, Scientific ReportNature, 2017, DOI: 10.1038/s41598-017-08841-x.

[3] Sousa PM, Gutiérrez Manuel, Mendoza Ernest, Llobera Andreu, Chu V, Conde JP, "Integration of Carbon Nanotubes into Electrostatically Actuated all-Polymer PEDOT: PSS/PMMA MEMS,” Procedia Engineering, 2011, vol. 25, pp. $1665-1668$.

[4] Tseng YT, Lin YC, Shih CC, Hsieh HC, Lee WY, Chiu YC, Chen WC, "Morphology and properties of PEDOT:PSS/soft polymer blends through hydrogen bonding interaction and their pressure sensor application," $J$. Mater. Chem. C. 2020, vol. 8, pp. 6013-6024.

[5] Nardes AM, Janssen RAJ, Kemerink M. "A Morphological Model for the Solvent-Enhanced Conductivity of PEDOT:PSS Thin Films," Advanced Functional Materials, 2008 vol. 18, iss. 6, pp. 865-871.

[6] Yazdimamaghani M, Razavi M, Mozafari M, Vashaee D, Kotturi H, Tayebi L. Biomineralization and biocompatibility studies of bone conductive scaffolds containing poly(3,4-ethylenedioxythiophene):poly(4-styrene sulfonate) (PEDOT:PSS). J. Mater. Sci: Materials in Medicine 2015, 26, 274. 